\title{
Multiple Sclerosis and Treatment: Drug Management and Nutrition with Exercise
}

\author{
Hector Melissinos \\ The Department of Biology, Athens College, Athens 15452, Greece
}

\begin{abstract}
MS (multiple sclerosis) is a chronic inflammatory disease of the CNS (central nervous system), characterized by the degeneration of the insulating caps of the brain and spinal nerve cells. Most axons are surrounded by an insulating lipoprotein, myelin, which helps in the transmission of nerve impulses. Myelin destruction disrupts the ability of parts of the nervous system to communicate, resulting in a wide range of symptoms, including physical, mental, and sometimes psychiatric problems. Multiple sclerosis affects more than 2.1 million people worldwide, but its incidence has increased in the last five decades among young people and women, with a 3: 1 ratio compared to men. Latitude is associated with a variety of environmental factors, most notably exposure to solar radiation. The risk of developing MS is inversely proportional to solar exposure and vitamin $\mathrm{D}$, particularly the $1,25-(\mathrm{OH})_{2} \mathrm{D}_{3}$ derivative that has anti-inflammatory and immunomodulatory activity, is probably the responsible protective agent. There are various therapies that are targeting the treatment of the disease, yet MS is considered an uncurable disease. Available medicines are aimed at relieving symptoms and improving the progression of the disease. In the present study, we will present the disease with reference to the pathogenesis and epidemiology of the disease. We will analyze the environmental factors that influence the development by reference to the etiology and pathophysiology, as well as reference to existing therapies. The ultimate aim of this study is to examine to what extent physical exercise and nutrition, in conjunction with pharmaceutical treatments and mainly intake of vitamin D, can contribute drastically to the fight against the disease and at the same time improve areas that make up the quality of life of the patient.
\end{abstract}

Key words: MS, CNS, myelin, etiology and pathophysiology of MS, vitamin D and MS.

\section{Introduction}

Sclerosis against myelin plaques or MS (multiple sclerosis) is an immune-mediated demyelinating disease of the human CNS (central nervous system). This is a chronic, progressive, inflammatory CNS disease, which mainly affects young adults, causing significant disability.

It is the leading cause of acquired neurological disability among 15-45 year-old people. In rare cases it occurs in children or older middle-aged people. Its aetiology is still unknown, making it difficult to cure the disease, despite the great progress made since the 1990s with the discovery of special drug therapies.

Clinically, the disease appears in 4 relatively distinct forms and based on these four categories can be distinguished as follows:

Corresponding author: Hector Melissinos, medical student, research fields: multiple sclerosis.
- RRMS (relapsing remitting multiple sclerosis): the most common form, as it affects about $85 \%$ of patients. It is characterized by recurrences or exacerbations of symptoms, followed by periods of remission, during which the symptoms improve or disappear.

- SPMS (secondary progressive multiple sclerosis): may develop in some patients with relapsing-intermittent disease. The course of the disease is continuously exacerbated with or without periods of remission or reduced symptom intensity. In several of these patients, agents that can modify the disease help delay the progression.

- PPMS (primary progressive multiple sclerosis): refers to approximately $10 \%$ of patients. Since the onset of the disease, the symptoms have gradually worsened, without recurrence or recession, perhaps sporadic periods of stability. This form is the most resistant to the usual treatments. 
- RPMS (relapsing progressive multiple sclerosis): rare form, refers to $5 \%$ of patients. Since its inception it has evolved with intermittent worsening intervals, without periods of recession [1].

As shown by extensive epidemiological studies, genetic factors play an important role. This means that it occurs in people who have some genetic predisposition, but this is triggered by an unknown environmental factor so far. Exposure or infection by some viruses, such as Epstein-Barr, low levels of vitamin D in the serum, smoking is suspected.

The disease is characterized by inflammatory foci that lead to the destruction of the myelin layer, a poly protein of three individual proteins, MBP, PLP, MOG, and axial lesions to varying degrees. The evolution of the disease varies greatly and is generally unpredictable.

The impact varies widely across the world and the highest rates are found in Europe, and North-European origin is the main risk factor, as well as in the America and South Australia. It is estimated that over 2.5 million people worldwide suffer from Multiple Sclerosis. As with other immune-mediated diseases, the female population prevails for most MS forms.

The effects of MS are significant and devastating due to the onset of the disease at an early age (average of 30 years), its chronic nature and the accumulation of physical disability. The inevitable results in the majority of patients are permanent neurological dysfunction and a decreased on average life expectancy due to an increased risk of complications associated with MS and advanced disability [2].

\section{Consequences of the Disease}

\subsection{Kinetic Disability in MS}

During the recurrent phase, the number of relapses contributes significantly to the aggravation of disability. More than 30\% of patients have moderate-to-severe spasticity, mainly in the legs. The initial clinical findings are often aesthetic disorders, with more common paraesthesia, dysaesthesia, diplopia, ataxia, vertigo and bladder sphincter disorders. Often numbness involves one foot, then progresses to the other and then rises to the pelvis, abdomen or chest. Although sensory disorders usually resolve, there are cases where they develop into chronic neuropathic pain. Trigeminal neuralgia may occur.

- Average walking time with mandatory use of a helmet (walking stick/crutch): 30 years.

- Average time for wheelchair restraint: 25 years for men and 30 for women.

\subsection{Other Disorders}

Optic neuritis may lead to partial or complete loss of vision.

Bladder disorders are in more than $90 \%$ of patients. One third of them experience at least one episode of incontinence per week.

Constipation is seen in 30\% of MS patients.

Fatigue, observed in $90 \%$ of patients and is the main cause of incapacity for work [3].

Sexual problems are often mentioned [2, 4].

\subsection{Impact on MS’s Patient Social Life}

It affects work and everyday activities, as well as family and social relationships.

\subsection{Mortality in MS}

- Reduce life expectancy from 7 to 14 years.

- Increase in suicide deaths by 7.5 times.

- About $50 \%$ of patients die of MS related causes.

\section{Epidemiology and Pathophysiology of Multiple Sclerosis}

MS is an autoimmune, inflammatory and neurodegenerative disease of the CNS, with multifactorial aetiology. The MHC (major histocompatibility complex) and its polymorphisms constitute the "golden standard" and the main genetic locus that affects the genetic framework of MS, but also the pathophysiology, prognosis, diagnosis, and therapeutic response to the disease. The association 
with the HLA-DRB1*1501 gene allele and its interactions with environmental factors such as vitamin $\mathrm{D}$ and Epstein-Barr virus or the estrogen receptor is now established.

Descriptive epidemiology continues to be the basis for understanding the interaction of genetic and environmental factors in the appearance of MS with its observations and correlations. The long latitude, as we move away from the equator, is associated with an increased incidence of MS, an association that has declined over the past decades. Latitude is associated with a variety of environmental factors, most notably, the exposure to solar radiation [2, 5-6].

The risk of developing MS is inversely proportional to sun exposure and vitamin D intake, which has anti-inflammatory and immunomodulatory effects, and is probably the responsible protective agent. This geographical observation excludes populations with a genetic susceptibility or protection against the disease, e.g. The Japanese, in whom the disease is uncommon and sometimes displays other clinical features.

In terms of sex, MS is more common in women, with the proportion of women: men at 2.5:1, and is constantly increasing in favor of women in the proportion of 5:1 in some populations. Concerning the factor of age, the incidence of the disease is low in childhood and increases after 18, with the highest incidence occurring in 20-40 year olds (mean age of about 30 years). Women are affected about 2-5 years earlier than men. At older ages the frequency decreases, and rarely occurs in individuals over 50 years of age when the frequency is equalized between the two sexes. In Europe, the prevalence rate of the disease is estimated at 83:100,000 and the mean annual incidence

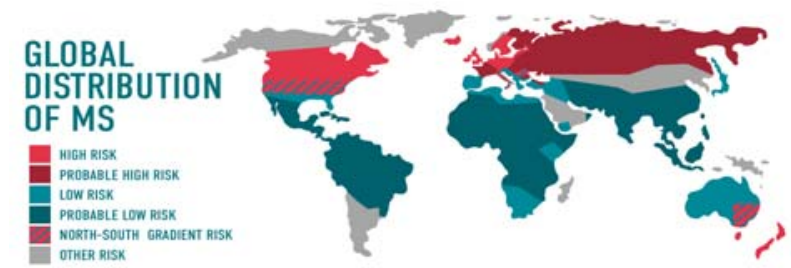

Fig. 1 Characterization of countries according to the likelihood of multiple sclerosis on a global scale in 1965. is $4.3: 100,000$.

The environmental factors that have been implicated at times in the onset of MS are various viral and bacterial infections, diet, exposure to animals, chemicals, metals, and also geographical and occupational factors. Especially with regard to responsible infectious agents, MS is an autoimmune disorder that can be triggered by a variety of microorganisms and viruses in genetically predisposed individuals. A typical example is the Epstein-Barr virus. In addition, people with a history of infectious mononucleosis have a higher risk of illness, especially if the infection occurred after puberty. Another interesting epidemiological observation is that in developed countries the incidence of the disease is increased, which is attributed to the lower incidence of childhood illnesses in these populations. A smaller exposure to common microbes in early life affects the maturation of the immune system and leads to the occurrence of autoimmune and allergic diseases (hypothesis of hygiene). Vaccines, such as the one of hepatitis $B$, have been implicated, but studies have not confirmed their causal relationship to the disease. Smoking also increases the risk of MS in a dose-dependent manner. In fact, studies suggest that there is a correlation between smoking and the risk of transition to Secondary Primary Disease and in general show worse prognosis. Finally, the timing of exposure to the various environmental risk factors plays an important role, with childhood and adolescence being the most important periods [2, 5-6].

MS is characterized by inflammatory lesions in the CNS. This neuropathology leads to specific losses in the myelin-oligodendrite complex as well as it leads to demyelination and degeneration of the neuro-axons. Although, as it mentioned above, the etiology of MS has not been yet elucidated, there are several indications that the disease originates from $\mathrm{T}$ lymphocytes that react against the myelin components. This hypothesis is supported by the presence of myelin reactive $\mathrm{T}$ lymphocytes, by the MS lesions. In models 
of PAH (a widely used animal model for MS), vaccination with myelin antigens or the transfer of sensitized $\mathrm{T}$ lymphocytes resulted in the production of MS-like features including highly dispersed demyelinating plaques and in perivascular inflammation. Demyelination and degeneration of the neuro-axons lead to the development of the characteristic lesions of MS in the brain, in spinal cord and in the optic nerves. Demyelination as well as the destruction of the neuro-axons is believed to occur when activated $\mathrm{T}$ lymphocytes penetrate the blood-brain barrier (AEF) and initiate an inflammatory reaction sequence. This inflammatory reaction sequence leads to endothelial cell activation, further recruitment of lymphocytes and monocytes and the release of pro-inflammatory cytokines. Cytokines interact with the immune system cells by enhancing the process. Activated $\mathrm{T}$ lymphocytes then recognize and attack the myelin proteins within the CNS. There is evidence that even in the early stages of the disease there is a destruction of the neuro-axons in the lesions with active inflammation. The degree of destruction of the neuro-axons is related to the extent of inflammation and the exposure of the neuro-axons that have undergone demyelination in the environment of the specific inflammation. There is also a loss of oligodendrocytes (myelin-producing cells) which prevent the re-myelination of neurons and reduce the production of nutrients that nourish the neurons and the axes. The clinical consequence of this process causes localized neurological "spaces", that is, incapability in the transmission of nerve impulses that occur prematurely in the course of the disease as well as neurological dysfunction, with increasing disability as the disease develops. Although the cause of the inflammatory sequence in MS is unknown, it is generally accepted that attachment and migration of inflammatory cells from the bloodstream through the $\mathrm{AEF}$ to the CNS are important, early stages in this specific process $[1,2,6-8]$.

\section{Mortality Rates and Survival Time}

Mortality rates vary from 0.5 to $3.6 / 100,000$ persons. Decline trends have been reported in some countries such as Denmark, Scotland, the Netherlands, Switzerland, Germany, Austria and Portugal. On the contrary, growth trends have been reported for countries such as Norway, Sweden, Bulgaria and in the female population of Italy.

\section{Therapeutic Approaches}

Multiple Sclerosis is considered as an incurable disease. The available medications aim at relieving the symptoms and, if possible, in improving the progression of the disease. They vary in safety and efficacy, so patients with MS have a number of restrictions. As a result, a large number of patients suffer a continuous worsening of their disease despite the appropriate treatment. Other patients cannot receive treatment due to specific contraindications, non-compliance with the approved indication criteria, interactions with other drugs they receive for cohorts, etc.

Available therapies include: interferons, selective immunosuppressants as well as treatments that act on the central nervous system. Interferons, being antiviral glycoproteins, the productions of which are triggered by the contamination of the organism by viral infections, act in an immune-modifying manner, limiting the morbidity of the disease and delaying its progression. They are divided into two broad categories: The type 1 interferons (IFN- $\alpha$, IFN- $\beta$ ) and the type 2 interferons (IFN- $\gamma$ ). IFN $\beta-1 \mathrm{a}$, known as Avonex, is administered intramuscularly once a week at a dose of $30 \mu \mathrm{g}$, IFN $\beta-1 \mathrm{a}$, known as Rebif, is administered subcutaneously three times a week at a dose of $44 \mu \mathrm{g}$ and IFN $\beta-1 \mathrm{~b}$, known as Betaferon, is injected subcutaneously every other day, at a dose of $0.25 \mathrm{mg}$. Various steroids or corticotropin were used to target immunosuppression. The first relatively effective 
immunosuppressant drug used was the ACTH, followed by other steroids, such as prednisol, and especially methylprednisolone, which is considered the most desirable drug for the treatment of relapses of the disease. Also, glatiramer acetate, also known as Copaxone, is used to try to mimic the basic protein of myelin. It is essentially about the acetate of a compound of synthetic polypeptides comprising four amino acids: L-alanine, L-glutamic acid, L-lysine and L-tyrosine. It is administered subcutaneously, on a daily basis, at a dose of $20 \mathrm{mg}$. Finally, Mitoxantrone, known as Noxantrone, is a synthetic antineoplastic agent which affects DNA and RNA, with possible immunosuppressive effects. It is administered at a dose of $5-12 \mathrm{mg} / \mathrm{m}^{2}$ of body surface, every three months. However, the dosage is limited to an administration over a time period of 2-3 years, or 120 to $140 \mathrm{mg} / \mathrm{m}^{2}$ of body surface cumulatively, due to induced cardiotoxicity $[1,2]$.

\section{The Role of Vitamin D in MS}

Vitamin D, which, among others, appears to have a strong effect on the mechanisms of the human body's defense system (immune system), and has been found to have been linked to protective action against diseases caused by immune system (Autoimmune diseases) such as multiple sclerosis and cancer.

According to a new study published in the journal Neurology, taking vitamin D supplements could be a safe and cost-effective treatment for people with multiple sclerosis. The researcher Dr. Peter A. Calabresi of the Johns Hopkins University School of Medicine in Baltimore and colleagues analyzed the effect of vitamin D supplements on 40 adults aged 18-55 years old with relapsing-intermittent multiple sclerosis.

Each participant received either 10,400 IU (high dose) or 800 IU (low dose) vitamin $\mathrm{D}_{3}$ supplements, daily for 6 months.

At the start of the study, at 3 and 6 months, blood levels of vitamin D were measured by each participant, and the researchers also evaluated $\mathrm{T}$ cell response, an indicator of disease activity.

The results revealed that people who received high doses of vitamin $\mathrm{D}_{3}$ showed a decrease in the percentage of $\mathrm{T}$ cells associated with disease activity. These cells are thought to play a role in the pathogenesis of multiple sclerosis. There was no reduction in the percentage of $\mathrm{T}$ lymphocytes associated with disease progression in the blood in those who received the low dose of vitamin $D_{3}$. According to the researchers, these findings suggest that there may be a promising new option for treating the disease. They see the results as exciting, as vitamin $\mathrm{D}$ are economical, safe and convenient treatments.

Further research is needed to confirm results in a larger group of people, but the findings are promising, they concluded.

It is noted that in people suffering from the disease, low levels of vitamin D have been associated with greater disability and more serious symptoms [9, 10].

\section{Could MS Be Treated with a Specific Nutrition or Diet? Is It a Reality or a Lie?}

Most doctors have rejected the idea that MS, a degenerative disease of the nervous system, may be linked to diet. It seems weird how such a serious and at the same time of unknown pathogenesis disease can be affected by something as simple as food. The medical community, instead of looking into eating habits for answers, expects a treatment for MS that comes from high-tech research, capable of identifying the primary cause in the immune system.

However, there is no conclusive evidence that diet has nothing to do with the cause or treatment of multiple sclerosis. In fact, scientific studies show diet as useful to improve the course of the disease.

MS is common in Canada, the US, northern Europe, but rarely occurs in Africa and Asia. Many studies have investigated the environmental factors that could account for the difference in the incidence of the disease among the different populations. The main 
factor seems to be the contact we have with our environment as well as the daily intake of food.

It appears that in the rich countries there is a higher incidence of the disease than in the less affluent countries, with the exception of Japan. Although the Japanese live in a modern, industrialized country with stress, pollution, smoking and all the habits that are common to other industrialized nations, they do not show the disease with the frequency that would be expected. The basis of diet in the Japanese is rice, a typical food consumed in poorer countries. The Japanese case provides strong indications that a diet rich in animal fats can set the foundation for triggering MS. Of course, diets rich in animal fats could cause several problems, but especially those containing dairy products, are more closely related to the development of the disease [11].

One theory suggests that cow's milk diet in infants sets the foundation for negative symptoms in the nervous system in later life. Cow's milk contains only $1 / 5$ linoleic acid (a necessary fatty acid) than there is in breast milk. Linoleic acid contributes to the formation of neural tissues. That's why children who grow up and in their diet have a high percentage of animal fats and a poor linoleic acid diet (like most children in our society) is likely to develop a weaker nervous system and to be prone to health problems as they grow older and get older.

Let's take a look at the health of people whose diet was limited to fat. During the Second World War, food was scarce and the stress was particularly high in occupied Western Europe. People could no longer afford to eat meat, so they turned to cereals and vegetables. The result was a dramatic reduction in the intake of animal products and total fat in their diet. Doctors observed that MS patients had 2 to 2.5 times fewer hospitalizations due to multiple sclerosis during the war.

A neurologist, Roy Swank, observed an improvement in MS disease when a patient follows a low-fat diet.
Three important findings have emerged from Dr. Swank:

- The sooner a MS patient adopts a low-fat diet, the better is the chance of avoiding further disease progression;

- Patients who restrict saturated fat intake to less than 20 grams per day have not shown the expected problems due to the disease (most Americans eat 125 grams or more every day);

- The 8 gram differences in daily saturated fat intake triples the death rate for people with MS [11].

\section{Conclusions}

The conclusions are clear. In order to fight the disease, there must be a "golden incision". Pharmaceutical treatment combined with balanced nutrition and potential exercise. Patients should follow the treatment recommended by the treating physician and then have to improve their eating habits so that they achieve the greatest possible effect on their treatment. From all the above we conclude that a good diet could be as low as possible in saturated fat, about $6 \%$ of total calories. This is translated into a low-fat, vegetarian diet with starchy foods, vegetables, fruits and foods that contain only 5 to $10 \%$ total fat [11].

The research's future is based on testing new pharmaceutical medications and drugs, particularly those acting in an immune-modifying way, while simultaneously further examining alternative factors, such as nutrition and exercise, which seem to reduce patients' symptoms as well as improve their quality of life. It is of the utmost importance that the factors that trigger the immune system in the first place, disorganize it and ultimately lead to the progress of the disease, are utterly defined.

\section{References}

[1] Luessi, F., Siffrin, V., and Zipp, F. 2012. "Neurodegeneration in Multiple Sclerosis: Novel Treatment Strategies.” Expert Review of Neurotherapeutics 12 (9): 1061-77.

[2] Calabresi, P. A. 2004. "Diagnosis and Management of Multiple Sclerosis.” Am. Fam. Physician 70: 1935-44. 
[3] Hauser, S. L., and Goodwin, D. S., 2008. "Multiple sclerosis and other demyelinating diseases," In Harrison's Principles of Internal Medicine, pp. 2611-21, McGraw-Hill Medical, New York, NY, USA, 17th edition.

[4] Noseworthy, J. H., Lucchinetti, C., Rodriguez, M., and Weinshenker, B. G. 2000. "Multiple Sclerosis." The New England Journal of Medicine 343 (13): 938-52.

[5] Ascherio, A., and Munger, K. L. 2007. "Environmental Risk Factors for Multiple Sclerosis. Part I. The Role of Infection (Review).” Ann. Neurol. 61: 288-99.

[6] Gabibov, A. G., Belogurov, A., Jr. Lomakin, Y. A., Zakharova, M. Y., Avakyan, M. E., Dubrovskaya, V. V., Smirnov, I. V., Ivanov, A. S., Molnar, A. A., Gurtsevitch, V. E., Diduk, S. V., Smirnova, K. V., Avalle, B., Sharanova, S. N., Tramontano, A., Friboulet, A., Boyko, A. N., Ponomarenko, N. A., and Tikunova, N. V. 2011. "Combinatorial Antibody Library from Multiple Sclerosis Patients Reveals Antibodies That Cross-React with Myelin Basic Protein and EBV Antigen." The Faseb
Journal 25 (12): 4211-21.

[7] Crespy, L., Zaaraoui, W., Lemaire, M., et al. 2011. "Prevalence of Grey Matter Pathology in Early Multiple Sclerosis Assessed by Magnetization Transfer Ratio Imaging.” PLoS One 6 (9): e24969.

[8] Navikas, V., and Link, H. 1996. "Review: Cytokines and the Pathogenesis of Multiple Sclerosis." J. Neurosci 45: 322-33.

[9] Ascherio, A., Munger, K. L., and Simon, K. C. 2010. "Vitamin D and Multiple Sclerosis." Lancet Neurol 9: 599-612.

[10] Sotirchos, E. S., Bhargava, P., Eckstein, C., et al. 2015. "Safety and Immunologic Effects of High- vs Low-Dose Cholecalciferol in Multiple Sclerosis." Neurology 86 (4): 382.

[11] McDougall John, A. 2010. "Treating Multiple Sclerosis with Diet: Fact or Fraud?" The Physicians Committee. N.p., 24 Sept. 2010. Web. 30 Dec. 2015. $<$ http://www.pcrm.org/health/health-topics/treating-multi ple-sclerosis-with-diet-fact-or>. 